\title{
Pharmacokinetics of Active Ingredients of Salvia miltiorrhiza and Carthamus tinctorius in Compatibility in Normal and Cerebral Ischemia Rats: A Comparative Study
}

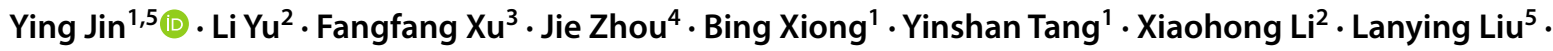 \\ Weifeng Jin $^{2}$
}

Published online: 11 December 2019

(c) The Author(s) 2019

\begin{abstract}
Background and Objective Dan-Hong injection, which comprises extracts of Salvia miltiorrhiza and Carthamus tinctorius, promotes blood circulation and reduces blood stasis. Combination of S. miltiorrhiza and C. tinctorius is more effective in treating cerebral ischemia than S. miltiorrhiza alone. This study aimed to examine the pharmacokinetic characteristics of four active ingredients of $S$. miltiorrhiza and $C$. tinctorius, namely danshensu (DSS), hydroxysafflor yellow A (HSYA), and salvianolic acid A (SAA) and B (SAB) in normal and cerebral ischemia rats.

Methods Normal and cerebral ischemia rats were injected via the tail vein with each active ingredient, and blood was collected through the jaw vein at different time points. The plasma concentration of the compatibility group was analyzed by high-performance liquid chromatography, and pharmacokinetic parameters were determined using Pharmacokinetic Kinetica 4.4 software.

Results The pharmacokinetics of the four active ingredients in the normal and cerebral ischemia rats were consistent with a two-compartment model. The area under the concentration-time curve was higher in normal rats than in cerebral ischemia rats, with a highly significant difference for SAA $(P<0.01)$. Clearance rates were lower in normal rats than in cerebral ischemia rats, with DSS showing the most significant difference $(P<0.01)$. Furthermore, there were significant differences between normal and cerebral ischemia rats in the distribution phase-elimination half life for DSS, SAA, and HSYA, as well as in the apparent volume of distribution for the central compartment for DSS and HSYA $(P<0.01)$. The plasma concentrations of the four active ingredients were higher in normal rats than in cerebral ischemia rats.

Conclusion Cerebral ischemia rats showed higher drug clearance rates and longer retention times than normal rats, which may be due to destruction of the blood-brain barrier during cerebral ischemia-reperfusion. The four active ingredients likely integrated and interacted with each other to affect target sites in the brain to protect against cerebral ischemic injury.
\end{abstract}

\section{Introduction}

Dan-Hong injection is composed of extracts of Salvia miltiorrhiza and Carthamus tinctorius, which has the effect of promoting blood circulation and reducing blood stasis. The main active ingredients of $S$. miltiorrhiza (Danshen in Chinese) include fat-soluble and water-soluble ingredients, among which the water-soluble ingredients are danshensu (DSS), salvianolic acid A (SAA), and salvianolic acid B (SAB) (Fig. 1)

Lanying Liu

bluelany@163.com

Weifeng Jin

jin_weifeng@126.com

Extended author information available on the last page of the article
[1]. The compatibility of these three active ingredients with hydroxysafflor yellow A (HSYA) in C. tinctorius (Honghua in Chinese) has a better therapeutic effect on cerebral ischemia [2-4]. SAA can scavenge free oxygen radicals $[5,6]$ and has a protective effect on cell damage caused by oxidative stress $[7,8]$. SAB not only has strong antioxidant effects [9-13], but it can also inhibit apoptosis [14-18]. DSS is an important water-soluble ingredient, which has been pharmacologically shown to improve blood circulation [19] and eliminate blood stasis [20,21], and can also protect against cerebral ischemia reperfusion injury [22]. HSYA can inhibit platelet aggregation $[23,24]$, reduce inflammatory response [25-28] and protect against myocardial ischemia $[29,30]$.

The active ingredients of both S. miltiorrhiza and $C$. tinctorius have obvious pharmacological effects, and there 


\section{Key Points}

The pharmacokinetic characteristics of Salvia miltiorrhiza and Carthamus tinctorius active ingredients were different in normal and cerebral ischemia rats

The reliability of the high performance liquid chromatography with diode array ultraviolet detection method for simultaneous determination of DSS, SAA, SAB, and HSYA in the plasma of normal and cerebral ischemia rats was fully validated by specificity, linear relationship, precision, recovery, and stability tests

are many studies examining the pharmacodynamic combinations of the active ingredients; however, there are few studies examining the pharmacokinetics of the active ingredients of S. miltiorrhiza and $C$. tinctorius in combination. The aim of this study was to establish a high-performance liquid chromatography with diode array ultraviolet detection (HPLC-DAD) method to examine the pharmacokinetic characteristics of the four above-mentioned active ingredients in both normal and cerebral ischemia rats, and to evaluate whether the pharmacokinetic properties in both normal and cerebral ischemia-reperfusion rats show any significant differences. The dosage of the four ingredients was from the results of our previous experiment [31].

\section{Materials and Methods}

\subsection{Chemicals and Reagents}

DSS (99\%, purity, SZ201707038DSS), SAA (99\%, purity, SZ201706001DA), SAB (99\%, purity, SZ201706003DB) and HSYA (99\%, purity, SZ201702005QA) were all obtained from Nanjing Shizhou Biology Technology Co., Ltd. (Nanjing, Jiangsu, China). Methanol and acetonitrile (chromatographic purity) were from Tedia Company $(\mathrm{OH}$, USA). Water was of ultra-pure quality while the remainder of the chemicals used were of analytical grade.
Fig. 1 Chemical structures of the four active ingredients. a Danshensu; b salvianolic acid A; c salvianolic acid B; d hydroxysafflor yellow A

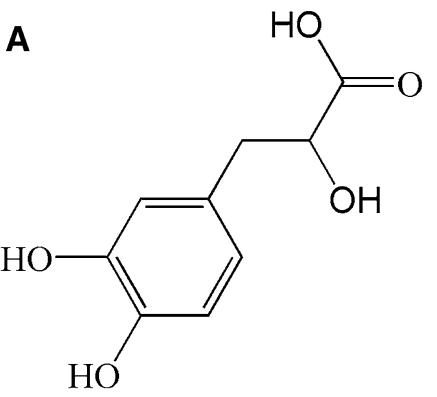<smiles>Cc1ccc(C[C@H](OC(=O)/C=C/c2ccc(O)c3c2[C@@H](C(=O)O[C@H](Cc2ccc(O)c(O)c2)C(=O)O)C(c2ccc(O)c(O)c2)O3)C(=O)O)cc1O</smiles>

B<smiles>O=C(/C=C/c1ccc(O)c(O)c1/C=C/c1ccc(O)c(O)c1)O[C@@H](Cc1ccc(O)c(O)c1)C(=O)O</smiles><smiles>O=C(/C=C/c1ccc(O)cc1)C1=C(O)[C@]2(O)C(O)=C([C@@H]3O[C@H](CO)[C@@H](O)[C@H](O)[C@H]3O)C(=O)C(O)=C2C(=O)C1=C(O)CO</smiles> 


\subsection{Animal Experiments}

Animal experiments were performed using adult male Sprague-Dawley (SD) rats (7-8 weeks, 280-300 g, Certification No. SCXK 2014-0001) which were purchased from Shanghai Laboratory Animal Center, Chinese Academy of Sciences. The rats were nurtured under standard conditions of humidity $(45 \pm 5 \%)$ and temperature controlled $\left(26 \pm 2{ }^{\circ} \mathrm{C}\right)$ with an alternating $12 \mathrm{~h}$ light/12 h dark cycle with freely available drinking water. The animals were nurtured for 7 days before surgery, and then fasted for $12 \mathrm{~h}$ with free access to water before surgery. All experimental procedures strictly followed the National Institutions of Health Guide for the Care and Use of Laboratory Animals.

\subsection{Instrumentation}

The following instruments were used in this study-an Agilent 1200 High Performance Liquid Chromatography System (including a G1311A Quaternary Gradient Pump, a G1316A Column Thermostat, a G1315D Diode Array Detector, a G1315D DAD Detector, a G1322A online degasser, and a chemical workstation; Waldbronn, Germany); a 2-16PK Centrifuge (Sigma, Germany); an XS205DU METTLER Precision Scale (Mettler Toledo); an ND100-1 Nitrogen Purger (Hangzhou Ruicheng Instrument Co., Ltd., Hangzhou, China); a QL-861 Vortex Mixer (Haimen Kylin-Bell Lab Instruments Co., Ltd., Haimen, China); an SK5210HP Ultrasonic Cleaner (Zhangjiagang Deke Ultrasound Co., Ltd., Zhangjiagang, China); a pure water meter (Millipore, Seattle, USA); and an SHB-IIIA Circulating Water Multipurpose Vacuum Pump (Henan Taikang Teaching Instrument Co., Ltd, Henan, China).

\subsection{Focal Cerebral Ischemia Model of Rats}

An improved rat model of middle cerebral artery occlusion (MCAO) using the thread embolization method, as reported by Longa et al., was used in this study [31]. Rats were intraperitoneally injected with $10 \%$ chloral hydrate $(4 \mathrm{~mL} / \mathrm{kg})$ as an anesthetic. An incision was then made in the median position of the neck and blunt dissections of the right common carotid artery (CCA), the external carotid artery (ECA), and the internal carotid artery (ICA) were performed. The CCA was ligated at the proximal end and the ECA root using a nylon thread and the ICA was closed using a miniature arterial clip. Another incision was made close to the bifurcation of the CCA and a nylon thread was inserted accordingly. The arterial clip was opened at the ICA end, and the line transited for approximately $20 \mathrm{~mm}$ until resistance could be felt before it was fixed. One hour later, the thread was gently extracted to finish the reperfusion. The incision was sutured and the rats returned to their cages. After recovery, Horner syndrome appeared in the right eye of the rats. The neurological symptoms of the rats were evaluated, and those with a score $\geq 2$ were selected as cerebral ischemia model animals.

\subsection{Grouping and Administration}

The rats were divided into a normal group and a model group $(n=5)$. In the model group, DSS $(30 \mathrm{mg} / \mathrm{kg})$, SAA $(10 \mathrm{mg} / \mathrm{kg})$, SAB $(8 \mathrm{mg} / \mathrm{kg})$, and HSYA $(4 \mathrm{mg} / \mathrm{kg})$ were injected into the tail vein immediately after $1 \mathrm{~h}$ of cerebral ischemia. The normal group underwent the same injections as the model group.

\subsection{Preparation of Reference Solution}

The reference standards for SAA, SAB, DSS, and HSYA were precisely weighed, and dissolved in methanol to obtain a $1 \mathrm{~g} / \mathrm{L}$ mother liquor. In parallel, a precise amount of $p$-hydroxybenzoic acid was weighed and dissolved in methanol to obtain a $1 \mathrm{~g} / \mathrm{L} p$-hydroxybenzoic acid solution as the internal standard mother liquor. The internal standard solution was diluted five-fold $(0.2 \mathrm{~g} / \mathrm{L})$ prior to use.

\subsection{Blood Sample Collection}

Before administration, the rats were fasted with free access to water for $12 \mathrm{~h}$. In the normal group, $0.5 \mathrm{~mL}$ of blank plasma was added to a test tube which contained $20 \mu \mathrm{L}$ of sodium heparin. The model group was administered drugs via tail vein injection, and $0.5 \mathrm{~mL}$ of blood was collected from the jaw vein [55] at 2, 5, 10, 15, 30, 45, 60, 90, 120, 180, 240, and $360 \mathrm{~min}$ after administration. Sodium heparin $(20 \mu \mathrm{L})$ was added as an anticoagulant. Samples were centrifuged at $3000 \mathrm{rpm}(825 \mathrm{~g})$ for $12 \mathrm{~min}$, and the supernatant was removed and stored at $-20{ }^{\circ} \mathrm{C}$.

\subsection{Plasma Sample Handling Methods}

Plasma samples $(100 \mu \mathrm{L})$ were transferred to a centrifuge tube containing $15 \mu \mathrm{L}$ of the internal standard solution $(0.2 \mathrm{~g} / \mathrm{L})$ after which $300 \mu \mathrm{L}$ of methanol was added. The samples were vortexed for $5 \mathrm{~min}$ and then centrifuged at $13,000 \mathrm{rpm}(15,493 \mathrm{~g})$ for $12 \mathrm{~min}$. The supernatant was removed and then dried under a nitrogen stream. The dried residue was reconstituted with $100 \mu \mathrm{L}$ of mobile phase and re-centrifuged at $13,000 \mathrm{rpm}(15,493 \mathrm{~g})$ for $12 \mathrm{~min}$, and the supernatant was used for HPLC analysis. 


\subsection{Chromatographic Conditions}

An Eclipse $\mathrm{XDB}-\mathrm{C}_{18}$ chromatographic column $(4.6 \mathrm{~mm} \times 250 \mathrm{~mm}, 5 \mu \mathrm{m})$ was used with a binary mobile phase system. Phase B was a $0.2 \%$ aqueous solution of phosphoric acid. The gradient elution consisted of 0-16 min, 90\% A-72\% A; 16-28 min, 72\% A-65\% A; 28-30 min, 65\% A-90\% A; A was $100 \%$ acetonitrile. The injection volume was $20 \mu \mathrm{L}$, the column temperature was $30^{\circ} \mathrm{C}$, the flow rate was $1.0 \mathrm{~mL} / \mathrm{min}$, and quantification was carried out by the internal standard peak area method. Chromatography was assessed using dual wavelengths-280 $\mathrm{nm}$ for SAA, SAB, and DSS and $403 \mathrm{~nm}$ for HSYA.
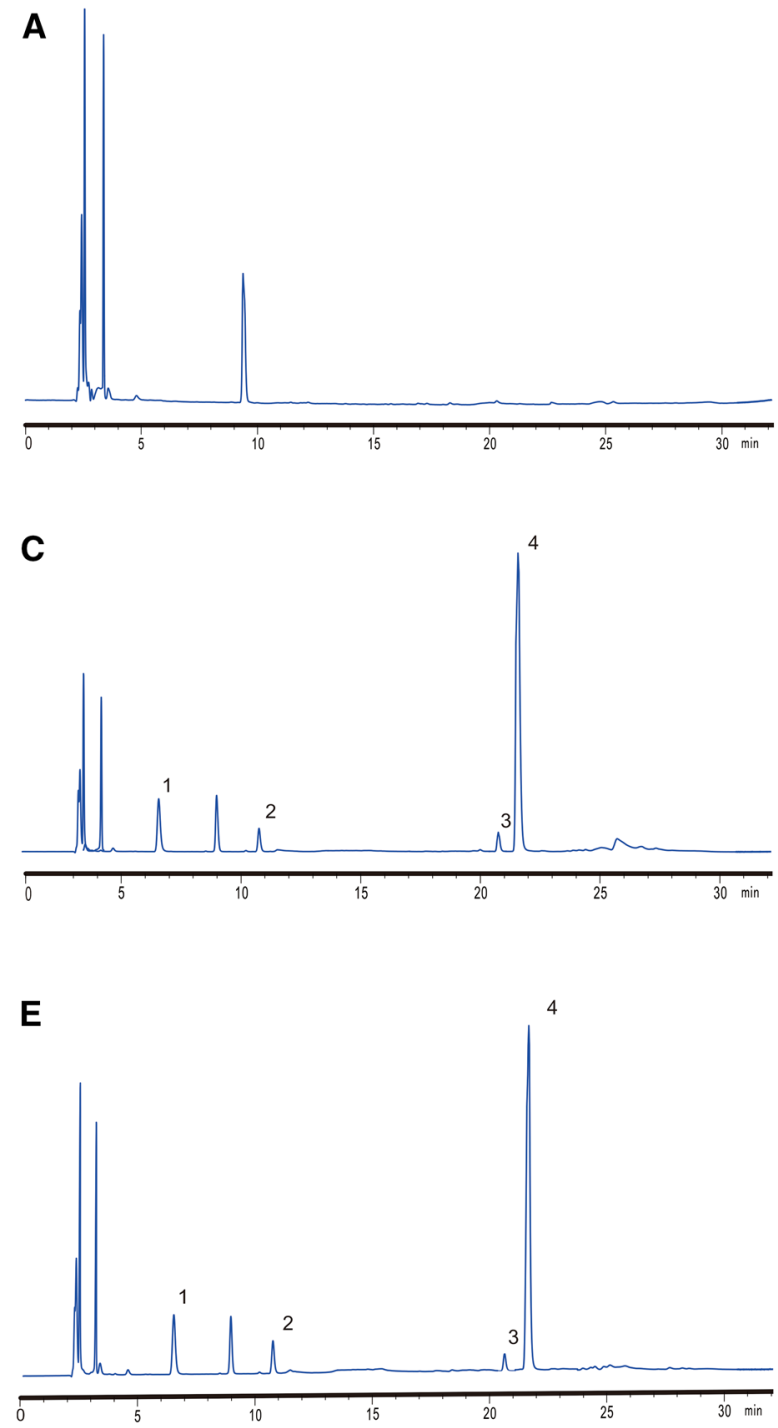

Fig. 2 HPLC chromatograms of rat plasma samples. a Blank plasma $280 \mathrm{~nm}$; b blank plasma $403 \mathrm{~nm}$; c blank plasma +internal standard + DSS + SAA + SAB $280 \mathrm{~nm}$; d blank plasma + internal standard + HSYA + SAA $403 \mathrm{~nm}$; e plasma samples from rats $10 \mathrm{~min}$ after drug administration + internal standard $280 \mathrm{~nm}$; f plasma sam-

\subsection{Data Analysis}

Pharmacokinetic parameters were calculated using a noncompartment model in software Kinetica version 4.4 (Innapase, Massachusetts, USA). Statistical comparisons were made between different groups using SPSS version 20.0 (SPSS Inc., Chicago, IL, USA) by an analysis of variance (ANOVA). All data were expressed as mean \pm standard deviation.

B
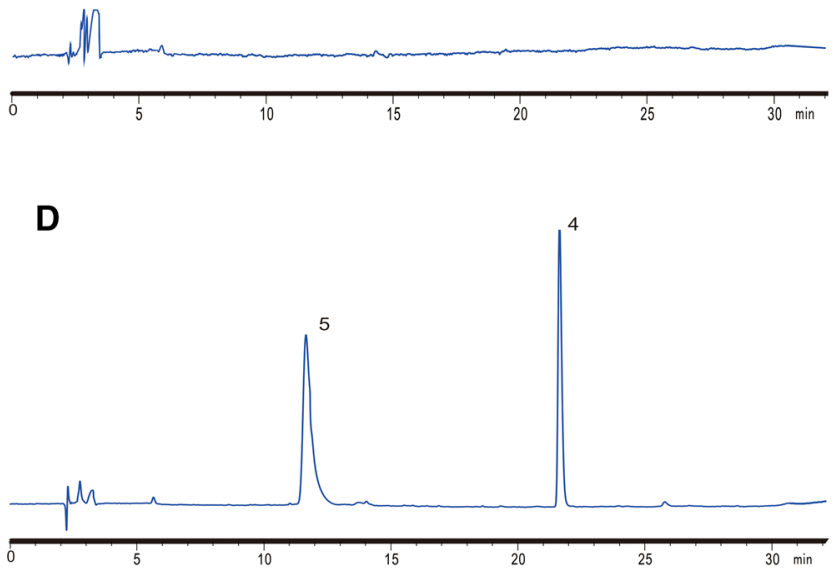

$\mathbf{F}$

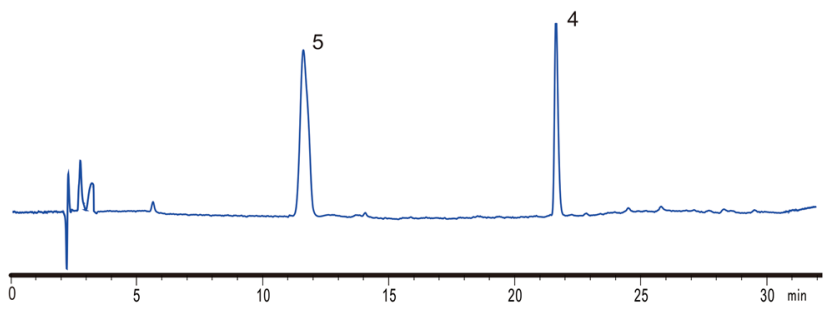

ples from rats $10 \mathrm{~min}$ after drug administration + internal standard $403 \mathrm{~nm}$. HPLC high-performance liquid chromatography, DSS danshensu, SAA salvianolic acid A, SAB salvianolic acid B, HSYA hydroxysafflor yellow A 


\section{Results}

\subsection{Specificity}

The treated blank plasma, mixed reference solution, and plasma samples were analyzed according to the abovementioned chromatographic conditions, as shown in Fig. 2. The results showed that under the selected chromatographic conditions, DSS, SAA, SAB, HSYA and the internal standard $p$-hydroxybenzoic acid could be completely separated from each other, had good peak shapes, and the endogenous peak impurities did not interfere with each other. These data indicated that the method had good specificity.

\subsection{Linear Range and Detection Limit}

The mixed reference solution was diluted with blank plasma to provide the following concentrations-DSS 100, 50, 25, $12.5,4$, and $0.5 \mu \mathrm{g} / \mathrm{mL}$; SAA $200,100,50,25,6.25$, and $0.5 \mu \mathrm{g} / \mathrm{mL}$; SAB $120,60,30,15,5$, and $0.5 \mu \mathrm{g} / \mathrm{mL}$; HSYA $60,30,15,7.5,4$, and $0.5 \mu \mathrm{g} / \mathrm{mL}$. The above-mentioned mixed reference plasma solutions were treated according to

Table 1 Linear relationships for each of the four ingredients

\begin{tabular}{lll}
\hline Active ingredients & Regression equations & $R^{2}$ \\
\hline SAA & $Y=0.123 X+0.1142$ & 0.9999 \\
SAB & $Y=0.0115 X+0.0694$ & 0.9974 \\
DSS & $Y=0.034 X+0.0301$ & 0.9995 \\
HSYA & $Y=0.088 X+0.0375$ & 0.9999 \\
\hline
\end{tabular}

DSS danshensu, SAA salvianolic acid A, SAB salvianolic acid B, HSYA hydroxysafflor yellow A the above-mentioned plasma sample handling methods and tested against the above-mentioned chromatographic conditions, and chromatograms and peak areas were recorded. The mass concentration of each drug in the plasma was plotted on the abscissa $(X)$, and the ratio of the peak area of the drug to the internal standard was plotted on the ordinate $(Y)$. The regression equations and correlation coefficients for the four ingredients were then obtained, as shown in Table 1. Based on a signal peak-to-noise ratio $=3$, the minimum detection limits were DSS $0.05 \mu \mathrm{g} / \mathrm{mL}$, SAA $0.04 \mu \mathrm{g} / \mathrm{mL}$, SAB $0.01 \mu \mathrm{g} / \mathrm{mL}$, and HSYA $0.02 \mu \mathrm{g} / \mathrm{mL}$. The calibration curve for DSS, SAA, SAB and HSYA were $Y=0.034 X+0.0301$ with $R^{2}=0.9995$ in the range of $0.5-100.0 \mu \mathrm{g} / \mathrm{mL}$, $Y=0.123 X+0.1142$ with $R^{2}=0.9999$ in the range of $0.5-200.0 \mu \mathrm{g} / \mathrm{mL}, Y=0.0115 X+0.0694$ with $R^{2}=0.9974$ in the range of $0.5-120.0 \mu \mathrm{g} / \mathrm{mL}$, and $Y=0.034 X+0.0301$ with $R^{2}=0.9999$ in the range of $0.5-60.0 \mu \mathrm{g} / \mathrm{mL}$, respectively.

\subsection{Precision and Recovery}

A low, medium, and high concentration of each ingredient that lay in the linear range of the standard curve was selected, and the corresponding standard plasma samples were prepared using blank plasma, treated according to the above-mentioned plasma sample handling methods and tested against the above-mentioned chromatographic conditions, and the chromatograms were obtained. The intraday precision was calculated over five consecutive runs in a single day. The interday precision was calculated over five consecutive days in a single week. The results are shown in Table 2. The intraday relative standard deviation (RSD) was $<3 \%(n=5)$ and the interday RSD was $<6 \%(n=5)$ using this analytical method, showing that the assay meets the requirements for biological sample analysis.

Table 2 Precision, recovery, and stability of the four active ingredients in rat plasma

\begin{tabular}{|c|c|c|c|c|c|}
\hline \multirow[t]{2}{*}{ Active ingredients } & \multirow{2}{*}{$\begin{array}{l}\text { Theoretical mass concentra- } \\
\text { tion }(\mu \mathrm{g} / \mathrm{mL})\end{array}$} & \multicolumn{2}{|c|}{ Precision RSD/\% } & \multirow[t]{2}{*}{ Recovery $\bar{X}_{ \pm s} / \%$} & \multirow{2}{*}{$\begin{array}{l}\text { Stability } \\
\text { RSD/\% }\end{array}$} \\
\hline & & Intraday (\%) & Interday (\%) & & \\
\hline \multirow[t]{3}{*}{ DSS } & 50 & 0.05 & 0.47 & $113.74 \pm 0.43$ & 0.25 \\
\hline & 25 & 0.31 & 0.35 & $110.15 \pm 0.50$ & 0.33 \\
\hline & 12.5 & 0.95 & 0.80 & $105.50 \pm 0.48$ & 0.37 \\
\hline \multirow[t]{3}{*}{ SAA } & 100 & 0.45 & 0.73 & $84.91 \pm 0.17$ & 0.53 \\
\hline & 50 & 0.54 & 0.30 & $78.86 \pm 0.71$ & 0.25 \\
\hline & 25 & 0.23 & 1.62 & $101.08 \pm 0.54$ & 0.08 \\
\hline \multirow[t]{3}{*}{$\mathrm{SAB}$} & 60 & 2.53 & 1.32 & $65.74 \pm 0.32$ & 3.62 \\
\hline & 30 & 0.45 & 4.39 & $105.19 \pm 2.60$ & 1.78 \\
\hline & 15 & 2.19 & 5.62 & $119.33 \pm 9.39$ & 1.59 \\
\hline \multirow[t]{3}{*}{ HSYA } & 30 & 0.29 & 0.72 & $93.01 \pm 0.30$ & 0.27 \\
\hline & 15 & 0.43 & 0.47 & $87.54 \pm 1.18$ & 0.33 \\
\hline & 7.5 & 0.78 & 0.44 & $91.41 \pm 0.31$ & 0.45 \\
\hline
\end{tabular}

$D S S$ danshensu, SAA salvianolic acid A, SAB salvianolic acid B, HSYA hydroxysafflor yellow A, RSD relative standard deviation 
The plasma samples containing low, medium, and high concentrations of the ingredients were prepared in blank plasma, and parallelized five parts for each mass concentration, and then subjected to the same treatment and test as those in the above-mentioned plasma sample handing methods and chromatographic conditions, respectively. The ratio of the peak area and internal standard for each measured ingredient was substituted into the regression equation to calculate the corresponding concentration in order to determine the recovery rate by comparing the average experimentally determined value with the theoretical value. The results are shown in Table 2.

\subsection{Stability}

Standard plasma samples were prepared containing the following concentrations of $\operatorname{DSS}(50,25,125 \mu \mathrm{g} / \mathrm{mL})$, SAA $(100,50,25 \mu \mathrm{g} / \mathrm{mL}), \operatorname{SAB}(60,30,15 \mu \mathrm{g} / \mathrm{mL})$, and HSYA $(30,15,7.5 \mu \mathrm{g} / \mathrm{mL})$. The plasma samples containing the different concentrations of drug were repeatedly frozen and thawed three times at $-20{ }^{\circ} \mathrm{C}$. The plasma samples were treated and tested with the same methods as in the above-mentioned plasma sample handling methods and chromatographic conditions. The RSDs for each ingredient sample after freeze thawing were DSS $0.25 \%$, $0.33 \%$, and $0.37 \%$; SAA $0.53 \%, 0.25 \%$, and $0.08 \%$; SAB $3.62 \%, 1.78 \%$, and $1.59 \%$; HSYA $0.27 \%, 0.33 \%$, and $0.45 \%$, for the respective concentrations listed above. These data indicated that sample stability after freezethawing was very good.

\subsection{Pharmacokinetics Study}

The plasma was treated as in the above-mentioned plasma sample handling methods and tested as in the abovementioned chromatographic conditions. Using the standard curve, the plasma concentration of the different drugs was determined at different time points. To elucidate the compatibility of the four active ingredients, their concentration-time curves were obtained by plotting the plasma drug concentration on the ordinate and time on the abscissa (Fig. 3). Following this, the pharmacokinetic parameters were calculated (Table 3 ).

Based on the concentration-time curves and the pharmacokinetic parameters, the pharmacokinetics of the four ingredients in the normal and cerebral ischemia rats were consistent with a two-compartment model. The compatibility of four different ingredients may influence the plasma concentrations and pharmacokinetic parameters.

The four ingredients showed statistically significant differences in absorption and distribution phases.

For DSS, compared with normal rats, there was a statistically significant difference $(P<0.01)$ in cerebral ischemia rats in the distribution phase-elimination half-life $\left(t_{1 / 2 \alpha}\right)$, the distribution volume of central compartment $\left(\mathrm{V}_{1}\right)$, the central compartment clearance $\left(\mathrm{CL}_{1}\right)$, the peripheral compartment clearance $\left(\mathrm{CL}_{2}\right)$, the primary clearance rate $\left(\mathrm{K}_{10}\right)$, and the central compartment transfer rate $\left(\mathrm{K}_{12}\right)$.

For SAA, compared with normal rats, there was an extremely significant difference $(P<0.01)$ in cerebral ischemia rats in $t_{1 / 2 \alpha}$, the area under the concentration-time curve $\left(\mathrm{AUC}_{(0-t)}\right)$, and $\mathrm{K}_{10}$; and a significant difference $(P<0.05)$ in $\mathrm{K}_{12}$, and the peripheral compartment transfer rate $\left(\mathrm{K}_{21}\right)$.

For SAB, compared with normal rats, there was a statistically significant difference $(P<0.01)$ in cerebral ischemia rats in $t_{1 / 2 \alpha}, \mathrm{V}_{1}$, the distribution volume of peripheral compartment $\left(\mathrm{V}_{2}\right), \mathrm{CL}_{1}, \mathrm{CL}_{2}, \mathrm{AUC}_{(0-t)}$, and $\mathrm{K}_{12}$.

For HSYA, compared with normal rats there was significant difference $(P<0.01)$ in cerebral ischemia rats in $t_{1 / 2 \alpha}$, $\mathrm{V}_{1}, \mathrm{~K}_{10}$, and $\mathrm{K}_{12}$.

The above pharmacokinetic models and parameters were selected according to the maximum $R^{2}$ value and the minimum Akaike's information criterion (AIC) value. The pharmacokinetic data were matched to one compartment, two compartments, and three compartments where the maximum $R^{2}$ value and the minimum AIC value were generated. Finally, a two-compartment pharmacokinetic model was selected. The results are shown in Table 4.

\section{Discussion}

The effect of treatment with Chinese medicines is often based on the interactions between multiple drug ingredients $[32,33]$. These ingredients interact with each other in multiple pathways, interacting with different targets [34, 35], providing fewer side-effects [36-38]. Traditional Chinese medicines can therefore provide new approaches and solutions in the treatment of diseases such as cerebrovascular disease. In recent years, the clinical application of traditional Chinese medicines has increased significantly [39-41]. One aspect of the use of such medicines that requires close attention is how to achieve synergy and greater effects but at low doses. Pharmacokinetics can play a key role in the evaluation of drug effects [42], drug-dosing intervals, etc., and is considered an efficient means of elucidating the mechanism of herbal formulas [43].

A Dan-Hong injection is composed of extracts of $S$. miltiorrhiza and $C$. tinctorius, which has the effect of promoting blood circulation and reducing blood stasis. It is often used for the treatment of cardio-cerebral-vascular disease caused by blood stasis [30, 44, 45]. Existing studies have shown that DSS, SAA, SAB, and HSYA have the ability to scavenge free oxygen radicals, inhibit apoptosis, and prevent inflammation [5-18, 25-28], thereby protecting against cerebral 

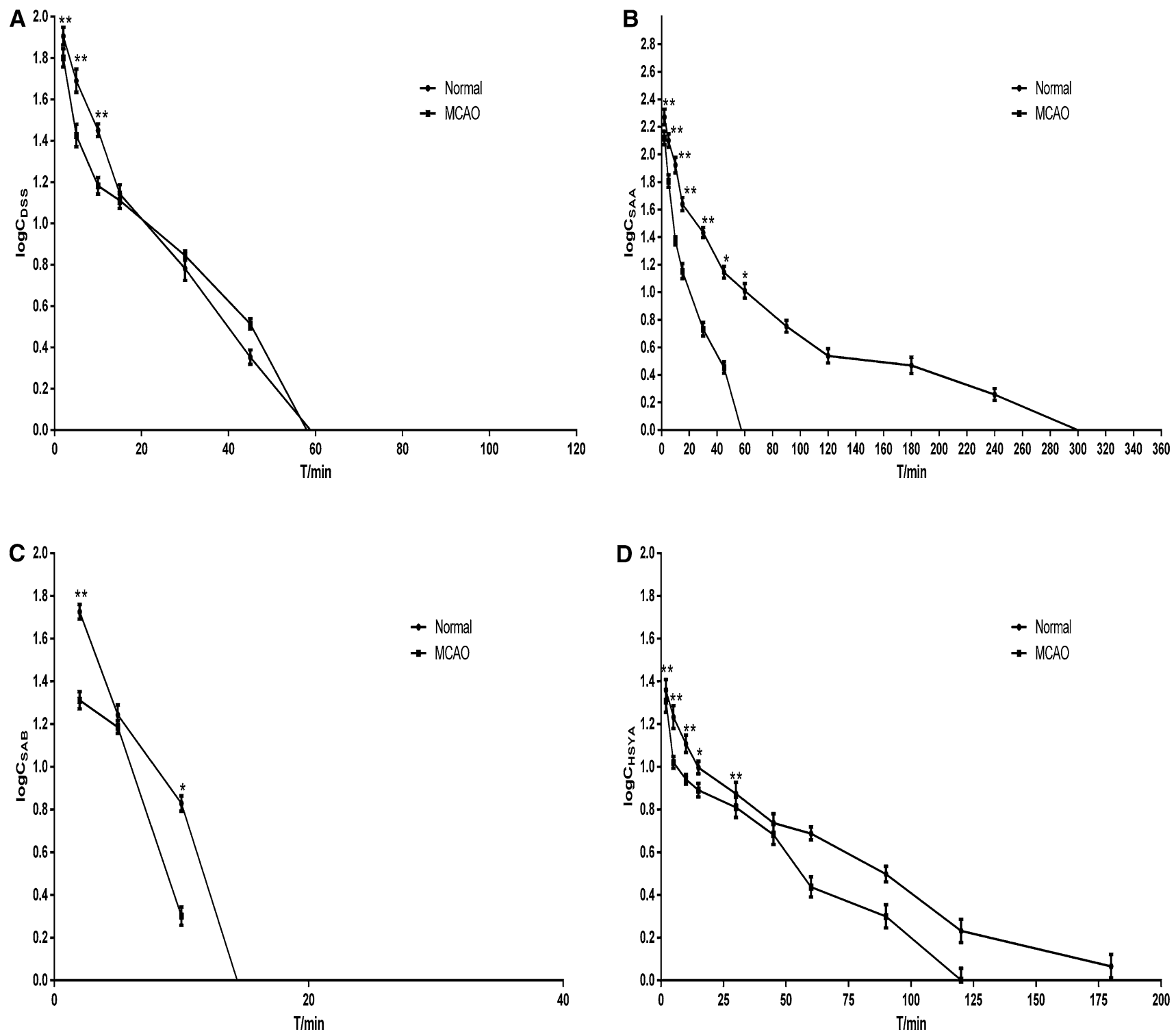

Fig. 3 The concentration $(\mu \mathrm{g} / \mathrm{mL})$ and time curves of the four active ingredients in compatibility in normal and cerebral ischemia rats. a Danshensu (DSS); b salvianolic acid A (SAA); c salvianolic

acid B (SAB); d hydroxysafflor yellow A (HSYA). $C$ concentration, $M C A O$ middle cerebral artery occlusion

ischemic injury. Over the past few years, the Dan-Hong injection and its ingredients have been widely studied in clinical and basic research [30, 46-48]. However, there are few studies evaluating the pharmacokinetic parameters of the active ingredients in the Dan-Hong injection.

Analysis of the components in the Dan-Hong injection and their pharmacodynamic effects has revealed that DSS, SAA, SAB, and HSYA are the main active ingredients [30, 46, 49-51]. Therefore, a pharmacokinetic study of the these four active ingredients appeared to be reasonable and necessary. In this study, we successfully created an HPLC method to determine the pharmacokinetic parameters for these four active ingredients and verified its sensitivity, specificity,

and selectivity. All analytes were found not to have any interference with the endogenous matrix. The plasma sample preparation process is simple and fast, simply requiring the precipitation of proteins with methanol, making it suitable for the analysis of mixed samples. Here, the HPLC method was successful in determining plasma pharmacokinetic parameters for DSS, SAA, SAB, and HSYA following time-course changes in both normal and cerebral ischemia rats [52-56]. We hope that this pharmacokinetic study can be used to fully elucidate the behavior of interaction after multicomponent compatibility, and provide a reference for clinical drug application. 


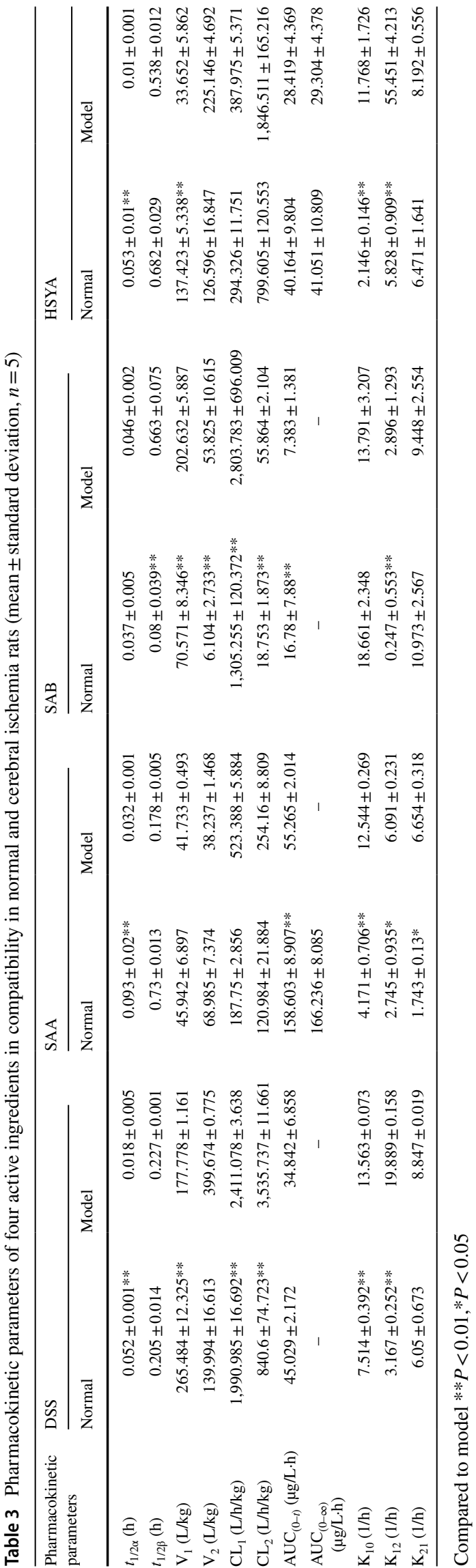

In the cerebral ischemia model group, due to severe damage of the vascular endothelial cells, vascular permeability increases, fluid leakage leads to blood stasis, blood flow is relatively obstructed, and plasma protein content may be lower than in normal rats, which affects the plasma protein rate of SAB [57] which, in turn, leads to an apparent distribution volume $\left(\mathrm{V}_{1}\right.$ and $\left.\mathrm{V}_{2}\right)$ in the central and peripheral chambers of the model group, which is larger than the normal group.

Metabolomics suggests that MCAO-induced cerebral ischemia is mainly caused by energy metabolism disorders, lipid metabolism disorders, inflammatory responses, and oxidative stress. The neuroprotective effects of SAB are associated with improved energy metabolism, lipid metabolism, inflammatory responses, and oxidative stress [58]. Therefore, we speculate that the clearance rate $\left(\mathrm{CL}_{1}, \mathrm{CL}_{2}\right)$ of the central and peripheral chambers of the model SAB group is increased on the basis of accelerating the metabolic reaction in the body when it produces the above improvement and neuroprotection. Meanwhile, the increase in the elimination phase half-life $\left(t_{1 / 2 \beta}\right)$ indicates that the elimination time and the drug action time are both prolonged in the body.

A large amount of SAA enters the central nervous system of rats with ischemia-reperfusion and promotes its protection and regulation of disorder metabolism [59]. We speculate that the $t_{1 / 2 \alpha}$ of the model SAA group is reduced because SAA promotes its distribution in the body when it enters the central nervous system to protect and regulate.

In addition, the blood barrier of cerebral ischemia rats will be damaged, and some drug components can have a small amount of permeability. Among then, SAB has been proved to improve mitochondrial function and play a neuroprotective role [60]. SAA can also participate in the protection and repair of the blood-brain barrier (BBB) [61]. Therefore, the $\mathrm{CL}_{1}$ and $\mathrm{CL}_{2}$ value of $\mathrm{SAB}$ in the plasma of the model group increased and the $t_{1 / 2 \alpha}$ value of SAA decreased compared with the normal group. HSYA can also repair BBB function [62]; HSYA could be detected in cerebrospinal fluid of cerebral ischemic model rats. Therefore, it was confirmed that HSYA can act on the brain through BBB, which explains the significant differences of HSYA V1 and $t 1 / 2 \alpha$ in the model group and normal group; the pharmacological action would be likely to promote the distribution and elimination of HSYA, and explains why the $\mathrm{V}_{1}$ and $t_{1 / 2 \alpha}$ of HSYA in the model group and normal group have significant differences.

A further review of the literature shows that cerebral ischemia reperfusion can affect renal function [63] and may have resulted in decreased renal reabsorption function, making the drug more easily excreted, thus leading to accelerated clearance rate. We speculated that the $t_{1 / 2 \alpha}$ of DSS in the rats in the model group and the significant difference in 
Table 4 Two-compartment model parameters for the four active ingredients in compatibility in normal and cerebral ischemia rats

\begin{tabular}{|c|c|c|c|c|c|c|c|c|}
\hline & \multicolumn{4}{|l|}{ Normal } & \multicolumn{4}{|l|}{ Model } \\
\hline & DSS & SAA & SAB & HSYA & DSS & SAA & SAB & HSYA \\
\hline AIC & 11.5374 & -7.0644 & 18.0474 & 13.5262 & 18.8572 & 16.4702 & 26.1806 & 13.8132 \\
\hline$R^{2}$ & 0.9974 & 0.9812 & 0.9378 & 0.9954 & 0.999 & 0.999 & 0.9416 & 0.9916 \\
\hline
\end{tabular}

$D S S$ danshensu, $S A A$ salvianolic acid A, SAB salvianolic acid B, HSYA hydroxysafflor yellow A clearance rate of $\mathrm{CL}_{1}$ and $\mathrm{CL}_{2}$ between rats in the normal group and the model group might be related to this.

Our study provides an innovative theory and good research ideas for the research of other proprietary Chinese medicines by detecting the pharmacokinetic characteristics of the active components of Dan-Hong injection in normal and cerebral ischemia rats and evaluating whether there are significant differences in pharmacokinetic properties in vivo. Simultaneously, the formula in our study plays an important role in the research on the creation of new drugs for the treatment of cerebral ischemic diseases, the improvement of dosage forms and the mechanism of prescription mechanism.

\section{Conclusion}

A HPLC-DAD method was established for simultaneous determination of DSS, SAA, SAB, and HSYA in the plasma of normal and cerebral ischemia rats. The reliability of this method was fully verified by specificity, linear relationship, precision, recovery, and stability tests. The main active ingredients of Danshen and Honghua in the plasma of normal and cerebral ischemia rats were quantitatively analyzed by this method. The pharmacokinetics of the tested ingredients in normal and cerebral ischemia rats were determined. The pharmacokinetics and characteristics of the tested ingredients were quite different in normal and cerebral ischemia rats. This study had certain reference value for studying the pharmacokinetics and characteristics of the main active substances of traditional Chinese medicine under pathological conditions.

Authors' Contribution YJ, LY, and FFX performed the rat model experiment and analyzed the data. YJ, LY, and JZ collected the data. BX, YST, and XHL performed the statistical analyses, and wrote the manuscript. LYL and WFJ designed and supervised the study, and edited the manuscript. All authors shared the raw data of this experimental study. All authors have contributed to and approved the final manuscript.

\section{Compliance with Ethical Standards}

Funding This study was supported by the Zhejiang Basic Public Welfare Research Project (Grant No. LGN19C190004), the China Postdoctoral Science Foundation (Grant No. 2018M630692), the Zhejiang Provincial Department of Education Research Project (Grant No. 201840159), and the University Visiting Scholar "Teacher Professional Development Project” (Grant No. FX2018022).
Conflict of Interest All authors have no conflict of interest to declare.

Ethical Approval The animal experiments adhered to the Zhejiang Chinese Medical University Animal Use Ordinance and were approved by the Ethics Committee of the Zhejiang Chinese Medical University.

Open Access This article is distributed under the terms of the Creative Commons Attribution-NonCommercial 4.0 International License (http://creativecommons.org/licenses/by-nc/4.0/), which permits any noncommercial use, distribution, and reproduction in any medium, provided you give appropriate credit to the original author(s) and the source, provide a link to the Creative Commons license, and indicate if changes were made.

\section{References}

1. Shi M, Huang FF, Deng CP, Wang Y, Kai GY. Bioactivities, biosynthesis and biotechnological production of phenolic acids in Salvia miltiorrhiza. Crit Rev Food Sci Nutr. 2019;59(6):953-64.

2. Zhao BX, Gu SF, Yong D, Shen MJ, Shen YQ. Solid lipid nanoparticles as carriers for oral delivery of hydroxysafflor yellow A. Int J Pharm. 2017;535(1-2):164-71.

3. Gao LN, Cui YL, Wang QS, Wang SX. Amelioration of Danhong injection on the lipopolysaccharide-stimulated systemic acute inflammatory reaction via multi-target strategy. J Ethnopharmacol. 2013;149(3):772-82.

4. Xu H, Liu WX, Liu TL, Su N, Guo C, Feng XN, et al. Synergistic neuroprotective effects of Danshensu and hydroxysafflor yellow A on cerebral ischemia-reperfusion injury in rats. Oncotarget. 2017;8(70):115434-43.

5. Zhang J, An SJ, Fu JQ, Liu P, Shao TM, Li ML, et al. Mixed aqueous extract of Salvia miltiorrhiza reduces blood pressure through inhibition of vascular remodelling and oxidative stress in spontaneously hypertensive rats. Cell Physiol Biochem. 2016;40(1-2):347-60.

6. Yuan TY, Chen YC, Zhang HF, Fang LH, Du GH. Salvianolic acid A, a component of Salvia miltiorrhiza, attenuates endothelial-mesenchymal transition of HPAECs induced by hypoxia. Am J Chin Med. 2017;45(6):1185-200.

7. Zu G, Zhou TT, Che NW, Zhang XW. Salvianolic acid A protects against oxidative stress and apoptosis induced by intestinal ischemia-reperfusion injury through activation of $\mathrm{Nrf} 2 / \mathrm{HO}-1$ pathways. Cell Physiol Biochem. 2018;49:2320-32.

8. Zhang H, Liu YY, Jiang Q, Li KR, Zhao YX, Cao C, et al. Salvianolic acid A protects RPE cells against oxidative stress through activation of Nrf2/HO-1 signaling. Free Radic Biol Med. 2014;69:219-28.

9. Wang YL, Zhang Q, Yin SJ, Cai L, Yang YX, Liu WJ, et al. Screening of blood-activating active components from DanshenHonghua herbal pair by spectrum-effect relationship analysis. Phytomedicine. 2019;54:149-58. 
10. Liu QM, Lu JY, Lin JR, Tang YL, Pu WL, Shi XG, et al. Salvianolic acid $B$ attenuates experimental skin fibrosis of systemic sclerosis. Biomed Pharmacother. 2019;110:546-53.

11. Zhang XD, Yu YG, Cen YS, Yang DF, Qi ZC, Hou ZN, et al. Bivariate correlation analysis of the chemometric profiles of Chinese wild Salvia miltiorrhiza based on UPLC-Qqq-MS and antioxidant activities. Molecules. 2018;23(3):538.

12. Chua LS, Lau CH, Chew CY, Ismail NIM, Soontorngun N. Phytochemical profile of Orthosiphon aristatus extracts after storage: rosmarinic acid and other caffeic acid derivatives. Phytomedicine. 2018;39:49-55.

13. Habtemariam S. Molecular pharmacology of rosmarinic and salvianolic acids: potential seeds for Alzheimer's and vascular dementia drugs. Int J Mol Sci. 2018;19(2):458.

14. Shoba E, Lakra R, Kiran MS, Korrapati PS. Strategic design of cardiac mimetic core-shell nanofibrous scaffold impregnated with salvianolic acid B and magnesium L-ascorbic acid 2 phosphate for myoblast differentiation. Mater Sci Eng C. 2018;90:131-47.

15. Yang K, Luo Y, Lu S, Hu RF, Du YY, Liao P, et al. Salvianolic acid $B$ and ginsenoside re synergistically protect against Ox-LDLinduced endothelial apoptosis through the antioxidative and antiinflammatory mechanisms. Front Pharmacol. 2018;9:662.

16. Gao S, Li SQ, Li Q, Zhang FY, Sun MQ, Wan ZL, et al. Protective effects of salvianolic acid B against hydrogen peroxideinduced apoptosis of human umbilical vein endothelial cells and underlying mechanisms. Int J Mol Med. 2019;44(2):457-68.

17. Zhai JH, Tao LN, Zhang YM, Gao H, Qu XY, Song YQ, et al. Salvianolic acid B attenuates apoptosis of HUVEC cells treated with high glucose or high fat via sirt1 activation. Evid Based Complement Altern Med eCAM. 2019;2019:9846325.

18. Yu XX, Guan QB, Wang YP, Shen HP, Zhai LP, Lu XD, et al. Anticonvulsant and anti-apoptosis effects of salvianolic acid $B$ on pentylenetetrazole-kindled rats via AKT/CREB/BDNF signaling. Epilepsy Res. 2019;154:90-6.

19. Huo MQ, Wang ZX, Wu DX, Zhang YL, Qiao YJ. Using coexpression protein interaction network analysis to identify mechanisms of Danshensu affecting patients with coronary heart disease. Int J Mol Sci. 2017;18(6):1298.

20. Tao L, Wang S, Zhao Y, Sheng XB, Wang AY, Zheng SZ, et al. Phenolcarboxylic acids from medicinal herbs exert anticancer effects through disruption of COX-2 activity. Phytomedicine. 2014;21(11):1473-82

21. Zheng XH, Zhao XF, Wang SX, Luo K, Wei YM, Zheng JB. Coadministration of Dalbergia odorifera increased biovailability Salvia miltiorrhizae in rabbits. Am J Chin Med. 2007;35(5):831-40.

22. Wei ZZ, Chen D, Liu LP, Gu X, Zhong W, Zhang YB, et al. Enhanced neurogenesis and collaterogenesis by sodium Danshensu treatment after focal cerebral ischemia in mice. Cell Transplant. 2018;27(4):622-36.

23. Sun Y, Xu DP, Qin Z, Wang PY, Hu BH, Yu JG, et al. Protective cerebrovascular effects of hydroxysafflor yellow A (HSYA) on ischemic stroke. Eur J Pharmacol. 2018;818:604-9.

24. Lu JX, Zhang CX, Hu Y, Zhang MH, Wang YN, Qian YX, et al. Application of multiple chemical and biological approaches for quality assessment of Carthamus tinctorius L. (safflower) by determining both the primary and secondary metabolites. Phytomedicine. 2019;58:152826.

25. Gao SQ, Chang C, Li JJ, Li Y, Niu XQ, Zhang DP, et al. Co-delivery of deferoxamine and hydroxysafflor yellow A to accelerate diabetic wound healing via enhanced angiogenesis. Drug Deliv. 2018;25(1):1779-89.

26. Wang T, Ding YX, He J, Ma CJ, Zhao Y, Wang ZH, et al. Hydroxysafflor yellow A attenuates lipopolysaccharide-induced neurotoxicity and neuroinflammation in primary mesencephalic cultures. Molecules. 2018;23(5):1210.
27. Zhang XH, Zheng W, Wang TR, Ren P, Wang FS, Ma XL, et al. Danshen-Chuanxiong-Honghua ameliorates cerebral impairment and improves spatial cognitive deficits after transient focal ischemia and identification of active compounds. Front Pharmacol. 2017;8:452.

28. Pei JP, Fan LH, Nan K, Li J, Dang XQ, Wang KZ. HSYA alleviates secondary neuronal death through attenuating oxidative stress, inflammatory response, and neural apoptosis in SD rat spinal cord compression injury. J Neuroinflammation. 2017;14(1):97.

29. Zhou DL, Ding TT, Ni B, Jing YY, Liu SX. Hydroxysafflor yellow A mitigated myocardial ischemia/reperfusion injury by inhibiting the activation of the JAK2/STAT1 pathway. Int J Mol Med. 2019;44(2):405-16.

30. Feng XJ, Li Y, Wang YN, Li LL, Little PJ, Xu SW, et al. Danhong injection in cardiovascular and cerebrovascular diseases: pharmacological actions, molecular mechanisms, and therapeutic potential. Pharmacol Res. 2019;139:62-75.

31. Chen YL, Wang HT, Zhou HF, Yu L, He Y, Li C, et al. Protective effects of Salvia miltiorrhiza and Carthamus tinctorius active ingredients in different compatibility on cerebral ischemia/reperfusion injury in rats. Chin Tradit Herb Drugs. 2018;49(16):3875-81.

32. Chen G, Xue WD, Zhu J. Full genetic analysis for genome-wide association study of Fangji: a powerful approach for effectively dissecting the molecular architecture of personalized traditional Chinese medicine. Acta Pharmacol Sin. 2018;39(6):906-11.

33. Shu Z, He W, Shahen M, Guo Z, Shu J, Wu T, et al. Clarifying of the potential mechanism of Sinisan formula for treatment of chronic hepatitis by systems pharmacology method. Biomed Pharmacother. 2018;100:532-50.

34. Lv J, Jia YT, Li J, Kuai WT, Li Y, Guo F, et al. Gegen Qinlian decoction enhances the effect of PD-1 blockade in colorectal cancer with microsatellite stability by remodelling the gut microbiota and the tumour microenvironment. Cell Death Dis. 2019;10(6):415.

35. Dong Y, Chen HW, Gao JL, Liu YM, Li J, Wang J. Bioactive ingredients in Chinese herbal medicines that target non-coding RNAs: promising new choices for disease treatment. Front Pharmacol. 2019;10:515.

36. Sharma R, Kabra A, Rao M, Prajapati P. Herbal and holistic solutions for neurodegenerative and depressive disorders: leads from ayurveda. Curr Pharm Des. 2018;24(22):2597-608.

37. Feng D, Ge C, Tan ZY, Sun JG, Xie Y, Yao L, et al. Isoflavones enhance pharmacokinetic exposure of active lovastatin acid via the upregulation of carboxylesterase in high-fat diet mice after oral administration of Xuezhikang capsules. Acta Pharmacol Sin. 2018;39(11):1804-15.

38. Naveed M, BiBi J, Kamboh AA, Suheryani I, Kakar I, Fazlani $\mathrm{SA}$, et al. Pharmacological values and therapeutic properties of black tea (Camellia sinensis): a comprehensive overview. Biomed Pharmacother. 2018;100:521-31.

39. Yu GH, Luo ZQ, Zhou YT, Zhang L, Wu Y, Ding L, et al. Uncovering the pharmacological mechanism of Carthamus tinctorius L. on cardiovascular disease by a systems pharmacology approach. Biomed Pharmacother. 2019;117:109094.

40. Miao LL, Zhou QM, Peng C, Liu ZH, Xiong L. Leonurus japonicus (Chinese motherwort), an excellent traditional medicine for obstetrical and gynecological diseases: a comprehensive overview. Biomed Pharmacother. 2019;117:109060.

41. Tsoi B, Chen X, Gao C, Wang S, Yuen SC, Yang D, et al. Neuroprotective effects and hepatorenal toxicity of Angong Niuhuang Wan against ischemia-reperfusion brain injury in rats. Front Pharmacol. 2019;10:593.

42. Liu RJ, Li X, Wei JY, Liu SB, Chang YY, Zhang JL, et al. A single dose of baicalin has no clinically significant effect on the 
pharmacokinetics of cyclosporine A in healthy chinese volunteers. Front Pharmacol. 2019;10:518.

43. Lin HM, Lin LF, Xia ZZ, Mao Y, Liu J, Xu LY, et al. Neuroprotective effects and UPLC-Q-TOF/MS-based active components identification of external applied a novel Wen-Luo-Tong microemulsion. Artif Cells Nanomed Biotechnol. 2018;46(8):1981-91.

44. Li C, Yang JH, Tong X, Zhao C, He Y, Wan HT. Precursor ion scan enhanced rapid identification of the chemical constituents of Danhong injection by liquid chromatography-tandem mass spectrometry: an integrated strategy. J Chromatogr A. 2019;1602:378-85.

45. Hu Z, Wang H, Fan GW, Zhang H, Wang XY, Mao JY, et al. Danhong injection mobilizes endothelial progenitor cells to repair vascular endothelium injury via upregulating the expression of Akt, eNOS and MMP-9. Phytomedicine. 2019;61:152850.

46. Xu WJ, Zhang YY, Yu YN, Li B, Liu J, Wang PQ, et al. Dosedependent target diversion of Danhong injection on the GluGLT-1/Gly-GlyRalpha dynamic balance module of cerebral ischemia. Pharmacol Res. 2018;135:80-8.

47. Orgah JO, Yu JH, Zhao TC, Wang LY, Yang MZ, Zhang Y, et al. Danhong injection reversed cardiac abnormality in brain-heart syndrome via local and remote beta-adrenergic receptor signaling. Front Pharmacol. 2018;9:692.

48. Fan HJ, Li M, Yu L, Jin WF, Yang JH, Zhang YY, et al. Effects of Danhong injection on platelet aggregation in hyperlipidemia rats. J Ethnopharmacol. 2018;212:67-73.

49. Li JP, Wang YY, Xu XJ, Cao WJ, Shen ZQ, Wang N, et al. Improved dialysis removal of protein-bound uremic toxins by salvianolic acids. Phytomedicine. 2019;57:166-73.

50. Zhao TC, Chang LY, Zhang BY, Lu M, Wang XY, Orgah JO, et al. Specific combination of salvianolic acids as core active ingredients of Danhong injection for treatment of arterial thrombosis and its derived dry gangrene. Front Pharmacol. 2017;8:361.

51. Li XX, Du FF, Jia WW, Olaleye OE, Xu F, Wang FQ, et al. Simultaneous determination of eight Danshen polyphenols in rat plasma and its application to a comparative pharmacokinetic study of DanHong injection and Danshen injection. J Sep Sci. 2017;40(7):1470-81.

52. Hui AL, Yin HY, Zhang Z, Zhou A, Chen JC, Yang L, et al. Enhancement of brain-targeting delivery of danshensu in rat through conjugation with pyrazine moiety to form danshensupyrazine ester. Drug Deliv Transl Res. 2018;8(3):787-96.

53. Wen XD, Wang CZ, Yu CH, Zhang ZY, Calway T, Wang YW, et al. Salvia miltiorrhiza (dan shen) significantly ameliorates colon inflammation in dextran sulfate sodium induced colitis. Am J Chin Med. 2013;41(5):1097-108.
54. Jiang XQ, Lv B, Li P, Ma XH, Wang T, Zhou Q, et al. Bioactivityintegrated UPLC/Q-TOF-MS of Danhong injection to identify NF-kappaB inhibitors and anti-inflammatory targets based on endothelial cell culture and network pharmacology. J Ethnopharmacol. 2015;174:270-6.

55. Yu L, Wan HF, Li C, Yang JH, Zhou HF, Wan HT, et al. Pharmacokinetics of active components from guhong injection in normal and pathological rat models of cerebral ischemia: a comparative study. Front Pharmacol. 2018;9:493.

56. Jia P, Wang SX, Meng X, Lan W, Luo J, Liao S, et al. Effects of ionic liquid and nanogold particles on high-performance liquid chromatography-electrochemical detection and their application in highly efficient separation and sensitive analysis of five phenolic acids in Xuebijing injection. Talanta. 2013;107:103-10.

57. Chen J, Wu H, Xu GB, Dai MM, Hu SL, Sun LL, et al. Determination of geniposide in adjuvant arthritis rat plasma by ultrahigh performance liquid chromatography tandem mass spectrometry method and its application to oral bioavailability and plasma protein binding ability studies. J Pharm Biomed Anal. 2015;108:122-8.

58. Duan WT, Wang L, Lv JZ, Gao K, Lu YQ, Qin SB, et al. Metabolomics study on the effects of salvianolic acid B and borneol for treating cerebral ischemia in rats by UPLC-Q/TOF-MS. Rejuvenation Res. 2019;22(4):313-24.

59. Feng SQ, Aa N, Geng JL, Huang JQ, Sun RB, Ge C, et al. Pharmacokinetic and metabolomic analyses of the neuroprotective effects of salvianolic acid A in a rat ischemic stroke model. Acta Pharmacol Sin. 2017;38(11):1435-44.

60. Jiang YF, Liu ZQ, Cui W, Zhang WT, Gong JP, Wang XM, et al. Antioxidant effect of salvianolic acid B on hippocampal CA1 neurons in mice with cerebral ischemia and reperfusion injury. Chin J Integr Med. 2015;21(7):516-22.

61. Zhang W, Song JK, Zhang X, Zhou QM, He GR, Xu XN, et al. Salvianolic acid A attenuates ischemia reperfusion induced rat brain barrier through MMP-9 inhibition and anti-inflammation. Chin J Nat Med. 2018;16(3):184-93.

62. Li LL, Wang N, Jin QZ, Wu Q, Liu YF, Wang Y. Protection of Tong-Qiao-Huo-Xue decoction against cerebral ischemic injury through reduction blood-brain barrier permeability. Chem Pharm Bull. 2017;65(11):1004-10.

63. Xu YM, Ding GH, Huang J, Xiong Y. Tanshinone IIA pretreatment attenuates ischemia/reperfusion-induced renal injury. Exp Ther Med. 2016;12(4):2741-6.

\section{Affiliations}

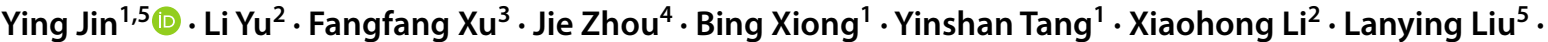 Weifeng Jin ${ }^{2}$}

Ying Jin

yingjin666@zju.edu.cn

$\mathrm{Li} \mathrm{Yu}$

yuli9119@126.com

Fangfang Xu

1178700326@zju.edu.cn

Jie Zhou

zhoujie0111@yeah.net
Bing Xiong

Xb5188@163.com

Yinshan Tang

2314038@zju.edu.cn

Xiaohong Li

li_xiaoh2005@163.com

1 Department of Rehabilitation in Traditional Chinese Medicine, The Second Affiliated Hospital, School 
of Medicine, Zhejiang University, No. 88 Jiefang Road, Hangzhou 310000, Zhejiang, China

2 School of Life Sciences, Zhejiang Chinese Medical University, No. 548 Binwen Road, Hangzhou 310053, Zhejiang, China

3 Department of Radiology, The Second Affiliated Hospital, School of Medicine, Zhejiang University, No. 88 Jiefang Road, Hangzhou 310000, Zhejiang, China
4 Department of Acupuncture, The Third Affiliated Hospital of Zhejiang, Chinese Medical University, No. 219 Moganshan Road, Hangzhou 310005, Zhejiang, China

5 Department of Acupuncture and Rehabilitation Medicine, Affiliated Hospital of Nanjing University of Chinese Medicine, No. 155 Hanzhong Road, Nanjing 210000, Jiangsu, China 